\title{
Experimental assessment of fracture of individual sand particles at different loading rates
}

Parab, Niranjan, nparab@purdue.edu; Claus, Benjamin; Hudspeth, Matthew; Sun, Jianzhuo; Chen, Weinong, Purdue University, United States; Fezzaa, Kamel; Xiao, Xianghui, Argonne National Laboratory, United States

\begin{abstract}
Failure mechanisms in individual sand particles under compressive loading at different loading rates were investigated using X-ray imaging. High speed X-ray phase contrast imaging was utilized to study the damage mechanisms in dry and wet sand under dynamic compressive loading. A modified Kolsky bar setup was used to apply controlled dynamic compression on two contacting sand particles. One of the particles was observed to pulverize, whereas other particle remained intact for dry sand particles with average failure load of $34.344 \mathrm{~N}$. In wet conditions, one of the particles was observed to break into large subparticles which pulverized upon further loading. Other particle was observed to stay intact. The failure load was observed to increase to $65.466 \mathrm{~N}$ for wet particles. 3D X-ray tomography was used to assess the failure of dry sand particles under static compressive loading. One particle broke into large subparticles which subsequently pulverized under static compressive loading. Even under static loading, second particle did not fail until first particle was completely pulverized. The pulverization load under static compressive loading was observed to be $42 \mathrm{~N}$. The order of pulverization for the particles was observed to be random in all experiments.
\end{abstract}

